\title{
Algebraic Models and the Correctness of Microprocessors
}

\author{
N. A. Harman and J. V. Tucker \\ Department of Computer Science, \\ University College of Swansea, \\ Swansea SA2 8PP, \\ WALES, \\ United Kingdom.
}

\begin{abstract}
A general algebraic method for modelling microprocessors at different levels of abstraction by means of iterated maps is introduced, supporting equational specification and design. We apply this iterated map method to the top level Programmer's Model specification, and to an abstract implementation, the Abstract Circuit Design. We formalise what it means for the implementation to correctly implement the specification. We illustrate the iterated map method with a case study.
\end{abstract}

\section{Introduction.}

In this paper we consider a general algebraic method for modelling microprocessors at different levels of abstraction, and for expressing formally the relationships between each level. These algebraic methods support the equational specification and design of microprocessors by a process of successive correctness preserving refinements. We consider models of microprocessors at different levels of abstraction, determined by time and by details of construction.

A clock is a means of dividing time into (not necessarily equal) segments; these segments are defined by steps or stages in a computation or process. We use this idea as follows. The method is to model a computer by means of the iteration of a map $f: A \rightarrow A$ in discrete time $T=\{0,1,2, \ldots\}$, defined by

$$
\begin{aligned}
& F: T \times A \rightarrow A, \\
& F(t, a)=f^{i}(a),
\end{aligned}
$$

for $t \in T, a \in A$. The set $A$ is made to model the state of the computer and the function $f$ to model the next-state map; thus $F(t, a)$ is the state of the computer at time $t \in T$ on operating from initial state $a \in A$. The computer is then modelled by the algebra

$$
(A, T \mid F) \text {. }
$$

The nature of the set $A$ of states and clock $T$ is determined by the level of abstraction of the computer. A typical clock would be the system clock. However, we may also consider instruction clocks, where each cycle represents the execution of a single instruction, or indeed any other division of time. Multiple clocks may exist in a single specification, and clocks may be formally related.

We show how to give an equational specification of this type of algebra using the fact that $F$ is defined by primitive recursive equations over the algebra

$$
(A, T \mid f, 0, t+1) \text {. }
$$

We show that this iterated map method is a systematic technique that decomposes the modelling of the computer into easily understood stages so that each model can be equationally specified using initial algebra semantics. 
We illustrate our algebraic tools by specifying a simple computer (based on the DEC PDP-8). We develop a specification at the programmer's level, and consider the implementation of this specification at the microcode level.

For work on formal methods in hardware, particularly those concerned with the formal specification and design of general-purpose computers, see Gordon [1983], Joyce [1987], and Stavridou [1993] which discuss the same simple computer, known as "Gordon's computer", Cohn [1987], Cullyer [1987a] and Cullyer [1987b] which discuss the Viper microprocessor, Graham [1990], Graham and Birtwistle [1990], and Birtwistle and Graham [1990] which discuss an implementation of Landin's SECD machine (Landin [1963]), Geser [1989], which discusses the Intel 8085 processor, and Hunt [1986]. For work on hardware specification and verification in general, see, for example, Gordon [1987], Cohn and Gordon [1990], Milne [1989], Milne [1990], Melham [1988], Subrahmanyam [1988], Johnson and Zhu [1991], and Zhu and Johnson [1991], and Weijland [1990].

This work is part of a project on the theory of verifiable synchronous concurrent algorithms, where a synchronous concurrent algorithm, or SCA, is an algebraic model of parallel deterministic computation. The work on formal specification, of which this is a part, is intended to support the work on SCAs by providing a basis for the formal verification of parallel systems modelled as SCAs. The work of the SCA group encompasses methodologies for specification and design; formal analysis; manipulation and verification of specifications and designs; and software tools. For further information see Tucker [1991] and Thompson and Tucker [1991]. For work on case studies, see Harman and Tucker [1988a], Harman and Tucker [1988b], Harman and Tucker [1989], Harman [1989], Harman and Tucker [1992]. In particular, Harman and Tucker [1988b] and chapter 7 of Harman [1989] are concerned with the specification of computers.

The structure of this paper is as follows. In $\S 2$ we discuss algebraic modelling and equational specification tools. In $\S 3$ we apply the iterated map algebras to model program execution and implementation in digital computers, and the relations between them. In $\S 4$ we outline the informal specification of a simple computer. In $\S 5$ we develop an algebraic specification of this computer. In $\S 6$ we propose an implementation of this computer. In $\S 7$, we consider the relationship between the specifications of the computer and of the controller, and show how the different levels of timing abstraction of each can be formally related.

The authors would like to thank J M Emmett for useful comments on a draft of this paper.

\section{Basic Algebraic Tools.}

In this section, we introduce the algebraic ideas for writing algebraic specifications of computers.

2.1. Abstract Data Types. The theory of modelling abstract data types by many-sorted algebras has been extensively covered in the literature: see, for example, Meinke and Tucker [1992], Wechler [1991] and Wirsing [1990].

A many-sorted algebra $A$ consists of one or more non-empty carrier sets $A_{1}, \ldots, A_{8}$, constants $c_{i} \in A_{s_{i}}$ from these sets, and operations $f_{i}$ of the form

$$
f_{i}: A_{s_{1}} \times \cdots \times A_{s_{n}} \rightarrow A_{s_{n+1}},
$$

where $i=1, \ldots, q$ and $s_{1}, \ldots, s_{n}, s_{n+1} \in\{1, \ldots, s\}$. 
2.2. Clocks. A clock $T$ is an algebra $(T \mid 0, t+1)$ where $T=\{0,1, \ldots\}$ identifies time intervals called clock cycles, 0 denotes the first clock cycle, and $t+1$ allows us to count clock cycles.

2.3. Iterated Maps and Primitive Recursion. Let $A$ be a non-empty set and $f: A \rightarrow A$, and form the algebra $(A \mid f)$. Let $T$ be a clock and consider the function

$$
F: T \times A \rightarrow A
$$

that iterates $f$ as follows: for $t \in T, a \in A$

$$
\begin{aligned}
& F(0, a)=a \\
& F(t+1, a)=f(F(t, a)) .
\end{aligned}
$$

A solution to this set $E_{F}$ of equations is

$$
F(t, a)=f^{t}(a)
$$

that generates the sequence, called an orbit,

$$
a, f(a), f^{2}(a), \ldots, f^{t}(a), \ldots
$$

and which will be used to represent the behaviour of a computer in time, starting from an initial state $a$.

The equations of $E_{F}$ are primitive recursive equations over the algebra $(A, T \mid f, 0, t+1)$, see Tucker and Zucker [1988], Tucker [1991].

2.4. A Decomposition. Consider the function $F: T \times A \rightarrow A$ as defined in $\S 2.3$. Let $A=A_{1} \times \cdots \times A_{n}$. Then the function $F$ can be rewritten

$$
F\left(t, a_{1}, \ldots, a_{n}\right)=\left(F_{1}\left(t, a_{1}, \ldots, a_{n}\right), \ldots, F_{n}\left(t, a_{1}, \ldots, a_{n}\right)\right)
$$

for $t \in T, a_{i} \in A_{i}$, and where $F_{i}: T \times A_{1} \times \cdots \times A_{n} \rightarrow A_{i}$ is the $i^{\text {th }}$ component function for $F, 1 \leq i \leq n$.

Furthermore, the function $f: A \rightarrow A$, whose iteration defines $F$, can be rewritten

$$
f\left(a_{1}, \ldots, a_{n}\right)=\left(f_{1}\left(a_{1}, \ldots, a_{n}\right), \ldots, f_{n}\left(a_{1}, \ldots, a_{n}\right)\right)
$$

for $a_{i} \in A_{i}$, and where $f_{i}: A_{1} \times \cdots \times A_{n} \rightarrow A_{i}$ is the $i^{\text {th }}$ component function for $f$.

Substituting these component functions for $F$ and $f$ we may rewrite the equations $E_{F}$ in $\$ 2.3$ as follows:

$$
\begin{aligned}
F_{1}\left(0, a_{1}, \ldots, a_{n}\right) & =a_{1}, \\
\vdots & \vdots \\
F_{n}\left(0, a_{1}, \ldots, a_{n}\right) & =a_{n} \\
F_{1}\left(t+1, a_{1}, \ldots, a_{n}\right) & =f_{1}\left(F_{1}\left(t, a_{1}, \ldots, a_{n}\right), \ldots, F_{n}\left(t, a_{1}, \ldots, a_{n}\right)\right), \\
\vdots & \vdots \\
F_{n}\left(t+1, a_{1}, \ldots, a_{n}\right) & =f_{n}\left(F_{1}\left(t, a_{1}, \ldots, a_{n}\right), \ldots, F_{n}\left(t, a_{1}, \ldots, a_{n}\right)\right) .
\end{aligned}
$$

Equations $E_{F_{1} \ldots F_{n}}$ are (simultaneous) primitive recursive equations over the algebra

$$
\left.\left(A_{1}, \ldots, A_{n}\right) \mid f_{1}, \ldots, f_{n}, 0, t+1\right) .
$$

See Tucker and Zucker [1993] 
2.4.1. Lemma. The following result is discussed further in Thompson and Tucker [1991] and Tucker and Zucker [1988]. Suppose that $\left(\Sigma_{0}, E_{0}\right)$ is an equational (or conditional equational) specification of

$$
\left(A_{1}, \ldots, A_{n} \mid f_{1}, \ldots, f_{n}\right)
$$

possibly containing hidden sets and functions. Then

$$
\left(\Sigma_{0} \cup \Sigma_{T} \cup\left\{F_{1}, \ldots, F_{n}\right\}, E_{0} \cup E_{F_{1}, \ldots, F_{n}}\right)
$$

is an equational (or conditional equational) specification of

$$
\left(A_{1}, \ldots, A_{n}, T \mid f_{1}, \ldots, f_{n}, 0, t+1, F_{1}, \ldots, F_{n}\right)
$$

and hence of

$$
\left(A_{1}, \ldots, A_{n}, T \mid F_{1}, \ldots, F_{n}\right)
$$

2.5. Comparing Iterated Maps. Consider the iteration of two maps $f: A \rightarrow$ $A$ and $g: B \rightarrow B$ defined by

$$
F: T \times A \rightarrow A \text { and } G: S \times B \rightarrow B
$$

where

$$
F(t, a)=f^{t}(a) \text { and } G(s, b)=g^{s}(b)
$$

for $t \in T, a \in A, s \in S$ and $b \in B$.

We compare these iterations as follows. First suppose there are maps

$$
\phi: A \rightarrow B \text { and } \psi: B \rightarrow A .
$$

that relate data. Then we say $G$ simulates $F$ with respect to $\phi$ and $\psi$ if there exists

$$
\bar{\lambda}: T \times A \rightarrow S
$$

such that the following diagram commutes.

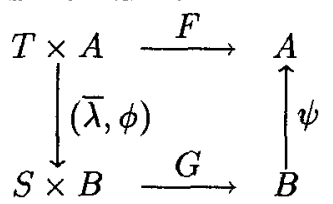

This means for $t \in T, a \in A$

$$
F(t, a)=\psi(G(\bar{\lambda}(t, a), \phi(a))),
$$

and, equivalently, given the definition of $F$ and $G, f^{t}(a)=\psi\left(g^{\bar{\lambda}(t, a)}(\phi(a))\right)$.

2.6. Retimings. Specifications may contain multiple clocks which are not equivalent in speed. Also, each cycle of a clock $T$ need not be the same length relative to another (e.g. standard) clock $R$ : that is, clocks can be irregular.

In order to relate multiple clocks we introduce retimings. A retiming $\lambda$ : $T \rightarrow R$ is a surjective, monotonic map between two clocks $T$ and $R$. We denote the set of retimings from clock $T$ to clock $R$ by $\operatorname{Ret}(T, R)$.

For each retiming $\lambda$ there is a corresponding immersion $\bar{\lambda}: R \rightarrow T$,

$$
\bar{\lambda}(r)=(\mu t \in T)[\lambda(t)=r] .
$$


The function $L:\left[T \rightarrow \mathbf{N}^{+}\right] \rightarrow \operatorname{Ret}(S, T)$ defines a retiming in which each clock cycle $L(x)(t)$ lasts $x(t)$ cycles of clock $S$. For a formal definition of $L$, see Harman [1989], or Harman and Tucker [1989]. Retimings may also be statedependent. That is, given an initial state $\sigma \in B$, we may define a retiming $\lambda: S \times B \rightarrow T$. Further formal tools can be developed from retimings: see Harman [1989], Harman and Tucker [1989], Harman and Tucker [1988a], and Harman and Tucker [1992].

\section{Computers as State Transformers.}

We will give computers state transformer or programmer's model specifications $P M$, that progressively update the state of the machine. By state, we mean those aspects of the internal structure of the computer visible to the programmer. That is, registers and memory that can be explicitly modified, sometimes called the architecture of the computer (Stallings [1987]). There is no requirement that these registers and/or memory actually exist in the form seen in $P M$ (though this is usually the case); however, the machine must behave as if they were present. In an actual design, there will be additional registers, hidden from the programmer, that are necessary for the implementation of the machine. These registers are not properly part of the specification. Additionally, we include memory in a state transformer specification even when specifying, say, a microprocessor, where memory is not an integral part of the device.

A hierarchy of specification models may be seen emerging. First, the programmer's model $P M$, followed by one or more levels modelling the implementation in successively greater detail.

3.1. Models and Abstraction in the Design of a Computer. The rôle of the programmer's model specification $P M$ is as part of a of specification of some implementation $I$, for which we may require a correctness proof. We can now ask: what is $I$, and how is it constructed?

In the case of a complex device the design would proceed in stages, with each successive stage a refinement of its predecessor, terminating in a set of chip masks, or other representation of the final hardware. We also admit the possibility of false trails in the design process: ideas that later are seen as inappropriate, and exploration of alternate designs. The design process will not be a simple trail of increasing detail: see Harman [1989], Harman and Tucker [1992]. In the basic case however, we can postulate an initial abstract circuit design $A C$, followed by one or more stages of detailed circuit design, and culminating in the final product: the chip mask-set, circuit board design, etc, whose characteristics are uniquely determined by a physical system.

In the case of a microprocessor, what does $A C$ consist of? We can partition a typical microprocessor into two main components: control and datapath. Additionally, since the initial programmer's model specification contained memory $M$, we must include $M$ in $A C$. However, we are not concerned with the design of $M$, and we can employ the same specification as used in $P M$. The abstract circuit design phase consists of specifying the control section $C T$ and the datapath $D P$, and composing thse together with $M$ to form $A C$. Then we must show that $A C$ correctly implements $P M$. We may continue the design process by subdividing $C T, D P$ and $M$ and specifying their components until we reach practical limits imposed by the physical behaviour of devices. 
3.2. Computer as an Iterated Map. The behaviour of a computer is described by an infinite sequence of states over time. The state comprises the contents of the machine's registers and memory. Additionally, there may be streams for input and output, but we will not consider these here. Let $C$ be the set of states. Starting from some initial state $\sigma \in C$, in which a program is held in memory and the program counter points to the start of this program, the machine state evolves in time as controlled by a next state function

$$
\text { comp : } C \rightarrow C \text {, }
$$

and a clock $T$. At every cycle $t$ of the clock, comp is applied to the current state $\sigma$ to generate a new state $\operatorname{com} p(\sigma)$. Hence the evolution of the state of the machine from time $0 \in T$ to time $t \in T$ is represented by

$$
\sigma, \operatorname{comp}(\sigma), \operatorname{comp}(\operatorname{comp}(\sigma)), \ldots, \operatorname{comp}^{t}(\sigma) \text {. }
$$

The machine is therefore represented by

$$
C O M P: T \times C \rightarrow C
$$

defined by

$$
\begin{aligned}
& \operatorname{COMP}(0, \sigma)=\sigma \\
& \operatorname{COMP}(t+1, \sigma)=\operatorname{comp}(\operatorname{COMP}(t, \sigma)) .
\end{aligned}
$$

However, it is often convenient to represent $C O M P$ in the form

$$
\operatorname{COMP}(t, \sigma)=\operatorname{comp}^{t}(\sigma) \text {. }
$$

We know from $\S 2.3$ that the computer is represented by a simultaneous primitive recursive function $C O M P$ over the algebra

$$
(C, T \mid \operatorname{comp}, 0, t+1)
$$

which we call the next-state algebra. The algebra

$$
(C, T \mid C O M P)
$$

we call the state algebra.

The speed of clock $T$ determines the level of timing abstraction of $C O M P$. Typically, $T$ will be the instruction clock, where each clock cycle represents the execution of a single instruction. Since instructions take differing amounts of "real" time to execute, the instruction clock will be irregular with respect to the system clock. The system clock is at a lower level of timing abstraction than the instruction clock. Each cycle of the system clock represents one cycle of the clock signal used to control the computer or microprocessor. In $\S 7$ we will show how to formally map from the instruction clock to the system clock.

3.3. A Decomposition. Following the ideas of $\S 2.4$, consider a decomposition of the state of a computer into the states of its CPU and memory. Let $C p u$ be the set of states of the central processing unit and $M e m$ be the set of states of the memory, and suppose that

$$
C=C p u \times M e m
$$

and hence for appropriate component functions

$$
C O M P(t, c, m)=(C P U(t, c, m), M E M(t, c, m))
$$


for all $t \in T, c \in C p u, m \in M e m$. If the next state function has component functions

$$
\operatorname{comp}(c, m)=(c p u(c, m), \operatorname{mem}(c, m))
$$

for $c \in C p u, m \in M e m$, then we may rewrite the equations for the computer.

$$
\begin{aligned}
& C P U(0, c, m)=c, \\
& M E M(0, c, m)=m, \\
& C P U(t+1, c, m)=\operatorname{cpu}(C P U(t, c, m), M E M(t, c, m)), \\
& M E M(t+1, c, m)=\operatorname{mem}(C P U(t, c, m), M E M(t, c, m)) .
\end{aligned}
$$

The next-state algebra becomes

$$
(C p u, M e m, T \mid c p u, m e m, 0, t+1),
$$

and the state algebra

$(C p u, M e m, T \mid C P U, M E M)$.

3.4. Machine Algebras. To specify a particular computer architecture at any level of abstraction, it is necessary to define the state set $C$ and next state function comp : $C \rightarrow C$. State set $C$ will normally be a cartesian product $C_{1} \times \cdots \times C_{n}$ where each $C_{i}, i=1, \ldots, n$ represents registers and memory: in particular, the component parts of $C p u$ and $\mathrm{Mem}$ (see $\$ 3.3$ ). The definition of comp then reduces to the definition of the coordinate functions $\operatorname{comp}_{i}: C_{1} \times$ $\cdots \times C_{n} \rightarrow C_{i}$, for $i=1, \ldots, n$.

The carriers $C_{1}, \ldots, C_{n}$ and the functions $\operatorname{com} p_{1}, \ldots, \operatorname{comp}_{n}$ of the state algebra are constructed from a machine algebra at a lower level of data abstraction. The machine algebra consists of carriers (typically vectors of bits) and functions representing ALU operations. A typical machine algebra for a microprocessor would be constructed as follows.

Let the algebra Bit consist of carrier $\{0,1\}$, constants 0 and 1 , and logical operations such as and, or, not, and so on (the precise choice depending on the architecture). Let $W_{n}=\{0,1\}^{n}$. The machine algebra $M$ is constructed by adding to $B i t$ carriers consisting of vectors of $B i t$, and special-purpose operations on these vectors: typically

$$
\left(B i t, W_{32}, W_{16}, W_{\mathrm{B}}, \mid \text { and }, \text { or, not, add, shift }, \text { sub, } m u l, \ldots\right),
$$

the precise choice again depending on the architecture.

3.5. Correctness. Given a programmer's model $P M$ of a computer, and an abstract circuit design $A C$ intended to implement $P M$, what conditions must be met if we are to say that $A C$ correctly implements $P M$ ?

The programmer's model specification $P M$ describes how the state $\sigma \in$ $C_{P M}$ of the machine evolves over time. Each component of $\sigma \in C_{P M}$ may, potentially, change during each cycle of clock $T$, where each cycle of $T$ represents one instruction. The execution of an instruction is determined by a next-state function pmcomp : $C_{P M} \rightarrow C_{P M}$, and so

$$
P M(t, \sigma)=p m \operatorname{comp} p^{t}(\sigma) \text {. }
$$

The abstract circuit design $A C$ behaves in a similar way, except now the state $\sigma^{\prime} \in C_{A C}$ is enlarged to include registers/memory required to implement 
$P M$, and the controlling clock system $S$ is faster than clock $T$. Again, computation is represented by a next-state function accomp: $C_{A C} \rightarrow C_{A C}$, and so

$$
A C\left(s, \sigma^{\prime}\right)=\operatorname{accomp} p^{\prime}\left(\sigma^{\prime}\right) .
$$

Although an element $\sigma^{\prime}$ of $C_{A C}$ will contain information not in $C_{P M}$, at the start of the execution of any instruction, that information will refer to the execution of the previous instruction. Any correct implementation of $P M$ will assume that, at the start of the execution of any instruction, those parts of $C_{A C}$ not in $C_{P M}$ contain "junk". Therefore, at the start of ally sequence of instructions we can assume that the contents of those parts of $\sigma^{\prime} \in C_{A C}$ not in $C_{P M}$ contain arbitary values.

Suppose there exists a function

$$
\alpha_{x}: C_{P M} \rightarrow C_{A C}
$$

which "pads" those elements of $C_{A C}$ not in $C_{P M}$ with values $x$, and furthermore, that there is a projection function $\pi: C_{A C} \rightarrow C_{P M}$, which projects out those parts of $C_{A C}$ also in $C_{P M}$. We may suppose that for all $\sigma \in C_{P M}$,

$$
\pi \alpha_{x}(\sigma)=\sigma .
$$

Informally then, $A C$ correctly implements $P M$ if, given an initial starting state $\sigma \in C_{P M}$, for any corresponding starting state $\alpha_{x}(\sigma) \in C_{A C}$, for any $x$, then for each cycle of clock $S$ corresponding with the start of a cycle of clock $T$, we can compute a time $\bar{\lambda}(t, \sigma)$, such that $\pi\left(A C\left(\bar{\lambda}(t, \sigma), \alpha_{x}(\sigma)\right)\right)$ is the state of $P M$ at clock cycle $t$.

We formalise the above correctness condition as follows. Given $P M: T \times$ $C_{P M} \rightarrow C_{P M}$ and $A C: S \times C_{A C} \rightarrow C_{A C}$, we require the following diagram to commute.

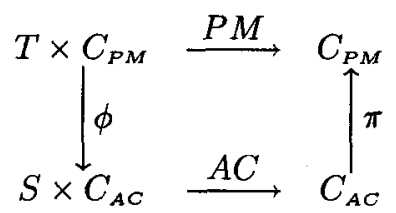

where $\phi: T \times C_{P M} \rightarrow S \times C_{A C}$ is defined by

$$
\phi(t, \sigma)=\left(\bar{\lambda}(t, \sigma), \alpha_{x}(\sigma)\right)
$$

$\alpha_{x}: C_{P M} \rightarrow C_{A C}$ "pads" $C_{P M}$ to $C_{A C}, \bar{\lambda}: T \times C_{P M} \rightarrow S$, and $\pi: C_{A C} \rightarrow C_{P M}$ projects out those parts of $C_{A C}$ also present in $C_{P M}$. Alternatively, we can express the correctness condition as follows: for all $\boldsymbol{x}$,

$$
P M(t, \sigma)=\pi\left(A C\left(\bar{\lambda}(t, \sigma), \alpha_{x}(\sigma)\right)\right) .
$$

Recall the definitions of $P M$ and $A C$ as the iterated maps

$$
\text { pmcomp : } C_{P M} \rightarrow C_{P M} \text { and accomp }: C_{A C} \rightarrow C_{A C}
$$

respectively. Then the condition for correctness becomes: for all $t \in T, \sigma \in C_{P M}$ and any padding values $x$

$$
p m \operatorname{comp} p^{t}(\sigma)=\pi\left(\operatorname{accomp} p^{\bar{\lambda}(\sigma, t)}\left(\alpha_{x}(\sigma)\right)\right) .
$$

Observe that $\bar{\lambda}: T \times C_{P M} \rightarrow S$ qualifies as the immersion of a state-dependent retiming $\lambda: S \times C_{P M} \rightarrow T(\S 2.6)$. 


\section{Informal Specification.}

The machine we will specify is based on the DEC PDP-8. This machine has been used previously as a design example: for instance, see Florentin [1991]. In this section, we will sketch the informal specification of the machine. Full informal specifications can be found in the literature: for example, Florentin [1991]. We will omit a number of straightforward features of the PDP-8 that are tedious to specify.

4.1. Registers, Word Size and Instruction Format. The PDP-8 uses a 12-bit word, giving an address space of $2^{12}(=4096)$ words. There is a single accumulator ACC, a single-bit link register $\mathrm{L}$, used for overflow detection and shifting, and a 12-bit program counter PC. All instructions are one word long, with format: 3-bit opcode; indirection bit; page bit; and 7-bit page offset. The page offset only allows 128 words to be addressed, hence the indirection and page-structured memory are used to allow access to the entire memory. Memory is divided into 128-word pages, and the page offset can either access a word in the first page of memory, or the page of memory containing the current instruction, depending on the page bit. The indirection bit allows indirect memory addressing.

4.2. Instruction Set. We will specify the following instructions.

(i) AND: Bitwise-and.

(ii) TAD: Two's complement Add.

(iii) ISZ: Increment and skip if zero.

(iv) DCA: Deposit and Clear ACC.

(v) JSR: Subroutine call.

(vii) JMP: Jump to the word specified by the memory operand.

Subroutine calls store the return address in the first word of the subroutine, and jump to the following word. To return, an indirect jump though the first word of the subroutine is necessary.

\section{Formal Specification.}

Recall from $\S 3.2$ and $\S 3.3$ our strategy for specifying a computer as an iterated map over a state set, and then decomposing this map into separate iterated maps for CPU and memory. We will construct a formal specification of the machine informally described in $\S 4$ in the manner of $\S 3.2$ and $\S 3.3$. We will not give a complete formal specification, but supply sufficient information for the reader in possession of an informal specification to complete the process.

Following $§ 3.2$ we may construct the state algebra

$$
(C, T \mid C O M P)
$$

of the machine from the algebra

$$
\text { (Cpu,Mem, T|CPU,MEM) }
$$

of the CPU and memory, with $C=C p u \times M e m$, as follows:

$$
\begin{aligned}
& C O M P: T \times C \rightarrow C, \\
& \operatorname{COMP}(t, c, m)=(C P U(t, c, m), M E M(t, c, m)) .
\end{aligned}
$$

In particular,

$$
\begin{aligned}
& C P U(0, c, m)=c, \\
& C P U(t+1, c, m)=c p u(C P U(t, c, m), M E M(t, c, m)),
\end{aligned}
$$


and

$$
\begin{aligned}
& M E M(0, c, m)=m, \\
& M E M(t+1, c, m)=\operatorname{mem}(C P U(t, c, m), M E M(t, c, m)) .
\end{aligned}
$$

To proceed, we must define the state sets $C p u$ and $M e m$, together with the next-state functions cpu and mem. There are two 12-bit registers ACC and PC, and a single-bit register $\mathrm{L}$, so we define

$$
C p u=A \times A \times B i t,
$$

where $A=W_{12}$. Memory consists of $2^{12} 12$-bit words. Hence we define

$$
\mathrm{Mem}=[A \rightarrow A] \text {. }
$$

Thus $C=C p u \times M e m=A \times A \times$ bit $\times[A \rightarrow A]$.

5.1. The Machine Algebra. Following the process outlined in $\S 3.4$, we define the machine algebra for our example. Our machine will require the following carriers. Let $A=W_{12}$ represent memory words and the accumulator. Let $L a=$ $W_{13}$ represent the result of additions. Additionally, we require $B i t$, the booleans $\mathbf{B}$ and the natural numbers $\mathbf{N}$, and the functions $\wedge: A^{2} \rightarrow A, \vee: A^{2} \rightarrow A$ and add : $A^{2} \rightarrow L a$.

In addition to ALU operations, we add the successor function for $\mathbf{N}$, together with some functions for manipulating bit vectors, converting bit vectors to $\mathbf{N}$, and performing memory substitution.

The field extraction functions bits $s_{j / l}^{W_{i}}: W_{i} \rightarrow W_{i}, j \geq i$, are defined by

$$
\operatorname{bits}_{j / l}^{W_{i}}\left(a_{1}, \ldots, a_{i}\right)=\left(0, \ldots, 0, a_{j}, \ldots, a_{l}\right)
$$

$0<j \leq l \leq i$.

The function $p n: A \rightarrow \mathbf{N}$ interprets a bit vector $\left(a_{1}, \ldots, a_{l}\right) \in A$ as a natural number, using the usual binary representation of positive integers. We will commonly, as an abuse of notation, omit applications of $p n$.

The function $s u b:[A \rightarrow A] \times A \times A \rightarrow[A \rightarrow A]$ is the memory substitution function, defined by

$$
s u b(m, a, l)(i)= \begin{cases}m(i), & \text { if } l \neq i \\ a, & \text { if } l=i\end{cases}
$$

We will use $m[a / l]$ to stand for $s u b(m, a, l)$. Summarising, the machine algebra is as follows:

$$
\begin{aligned}
& (B i t, A, L a, A \rightarrow A, \mathbf{B}, \mathbf{N} \mid \wedge, \vee, \text { add }, \text { succ, } \\
& \text { bits } \left._{\mathbf{j} / i}^{W_{i}}, \text { pn, sub, cases, }=\right)
\end{aligned}
$$

Observe that we can use $b i t s_{j / i}^{W_{i}}$ to construct projection functions.

5.2. Field Extraction Functions. We define the following functions to extract the various fields of an instruction. The opcode field is extracted by op $: A \rightarrow \mathrm{N}$, defined by

$$
o p(a)=p n\left(\text { bits }_{1 / 3}^{A}(a)\right) .
$$

We use $p n$ to convert the three opcode bits into a natural number. This makes the specification easier to read.

The page offset field is extracted by pgoff : $A \rightarrow A$, defined by

$$
\operatorname{pgoff}(a)=\operatorname{bits}_{6 / 12}^{A}(a) \text {. }
$$


The indirection bit is extracted by indir : $A \rightarrow \mathbf{B}$, defined by

$$
\operatorname{indir}\left(a_{1}, \ldots, a_{12}\right)= \begin{cases}t t, & \text { if } a_{4}=1 ; \\ f f, & \text { if } a_{4}=0 .\end{cases}
$$

The page bit is extracted by pgbit $: A \rightarrow \mathbf{B}$, defined by

$$
\operatorname{pgbit}\left(a_{1}, \ldots, a_{12}\right)= \begin{cases}t t, & \text { if } a_{5}=1 ; \\ f f, & \text { if } a_{5}=0 .\end{cases}
$$

5.3. Next-State Functions. First we define the CPU next state function

$$
\text { cpu }: A \times A \times B i t \times[A \rightarrow A] \rightarrow A \times A \times B i t
$$

as follows:

$$
\begin{aligned}
& c p u(a, p c, l, m)= \\
& \begin{cases}(a \wedge \text { mval }(p c, m), p c+1, l), & \text { if } o p(m(p c))=0 ;(i) \\
\left(b i t s_{1 / 12}^{L a}(a d d(a, l, m v a l(p c, m))), p c+1,\right. & \text { if } o p(m(p c))=1 ;(i i) \\
\left.a d d_{13}(a, l, m v a l(p c, m))\right) & \text { if } o p(m(p c))=2 ;(i i i) \\
(a, t s k i p(p c, m), l) & \text { if } o p(m(p c))=3 ;(i v) \\
(0, p c+1, l), & \text { if } o p(m(p c))=4 ;(v) \\
(a, m v a l(p c, m)+1, l), & \text { if } o p(m(p c))=5 .(v i) \\
(a, m v a l(p c, m), l), & \end{cases}
\end{aligned}
$$

We explain each case briefly below.

(i) AND: the contents of $a$ are replaced with the bitwise and of the initial value of $a$ and the memory operand mval $(p c, m)$. The program counter $p c$ is incremented.

(ii) TAD: accumulator $a$ is replaced by the least-significant 12 bits of the sum of $a$ and mval, and $l$ is replaced by the most significant bit of this sum. The program counter $p c$ is incremented.

(iii) ISZ: the program counter is incremented by one or two (by tskip), depending on the new value of the memory word.

(iv) DCA: the accumulator is set to zero. The program counter is incremented.

(v) JSR: the value of $p c$ is replaced by mval+1.

(vi) JMP: the value of $p c$ is replaced by mval.

Next we define the memory next-state function

$$
\text { mem }: A \times A \times B i t \times[A \rightarrow A] \rightarrow[A \rightarrow A]
$$

as follows:

$$
\begin{aligned}
& m e m(a, p c, l, m)= \\
& \begin{cases}m[\operatorname{mval}(p c, m)+1 / \operatorname{maddr}(p c, m)], & \text { if } o p(m(p c))=2 ;(i) \\
m[a / \operatorname{madd}(p c, m)], & \text { if } o p(m(p c))=3 ;(i i) \\
m[p c+1 / \operatorname{mad} r(p c, m)], & \text { if } o p(m(p c))=4 ;(i i i) \\
m, & \text { otherwise. }\end{cases}
\end{aligned}
$$

We explain each case briefly below.

(i) ISZ: the memory word indicated by maddr is incremented by one.

(ii) DCA: the accumulator $a$ is stored at memory word maddr.

(iii) JSR: the program counter $p c$ plus one is stored at memory word maddr.

For all other instructions, memory is not changed. It now remains to define the subfunctions of comp. 
5.4. Memory Access. We need both memory address (maddr) and read memory (mval) functions. We define maddr:A×Mem $\rightarrow A$ as follows:

$$
\begin{aligned}
& \operatorname{maddr}(p c, m)= \\
& \begin{cases}p g \circ f(m(p c)), & \text { if } p g \operatorname{bit}(m(p c))=0 \text { and } \operatorname{indir}(m(p c))=0 ;(i) \\
p g \circ f f(m(p c)) \vee & \text { if } p g b i t(m(p c))=1 \text { and } \operatorname{indir}(m(p c))=0 ;(i i) \\
p c \wedge A D D R M S K, & \\
m(p g \circ f(m(p c))), & \text { if } p g b i t(m(p c))=0 \text { and } \operatorname{indir}(m(p c))=1 ;(i i i) \\
m(p g \circ f(m(p c)) \vee & \text { if } p g b i t(m(p c))=1 \text { and } \operatorname{indir}(m(p c))=1 .(i v) \\
(p c \wedge A D D R M S K)), & \end{cases}
\end{aligned}
$$

where $A D D R M S K=(1,1,1,1,1,0,0,0,0,0,0,0)$. In cases $(i)$ and $(i i i)$ we are accessing memory words in page zero. In cases (ii) and (iv) we are accessing memory words in the current page, and so must extract the most significant five bits of $p c$ and use them to extend the page offset. In cases (iii) and ( $i v)$, we are using indirect addressing, and so must make a memory access.

We define mval : $A \times M e m \rightarrow A$ as follows.

$$
\operatorname{mval}(p c, m)=m(\operatorname{maddr}(p c, m)) \text {. }
$$

We define tskip : $A \times M \rightarrow A$ as follows.

$$
\operatorname{tskip}(p c, m)= \begin{cases}p c+1, & \text { if } \operatorname{mval}(p c, m)+1 \neq 0 \\ p c+2, & \text { if } \operatorname{mval}(p c, m)+1=0\end{cases}
$$

5.5. The Programmer's Model. We now compose $C P U$ and $M E M$ to construct the programmer's model specification COMP. Recall from $\S 2.5$ and $\S 3.3$ that

$$
\operatorname{COMP}(t, c, m)=(C P U(t, c, m), M E M(t, c, m))
$$

and

$$
\operatorname{comp}(c, m)=(c p u(c, m), \operatorname{me} m(c, m)) \text {. }
$$

By using (1) and (2), expanding our definitions of $C P U$ and $M E M$, and substituting appropriately, we can construct

$$
C O M P(t, c, m)=c o m p^{t}(c, m),
$$

where comp is defined in (2).

5.6. Algebraic Structure of a Microprocessor. It is easy to check that function comp is polynomial over the machine algebra, and that therefore COMP is primitive recursive over the machine algebra. Since the machine algebra is easily algebraically specified, the state algebra of the computer is algebraically specified by lemma 2.4.1. In fact, the specification can be chosen to have useful term rewriting properties (e.g. orthogonality and completeness).

\section{A Microprogrammed Implementation.}

We now consider how $C O M P$ specified in $\$ 5$ may be implemented. We will proceed in the manner outlined in $\S 3.1$. First, we will decide on, and informally specify, an appropriate datapath $D P$, and controller $C T$. Then we will show how $D P$ and $C T$ may be formalised as iterated maps. 


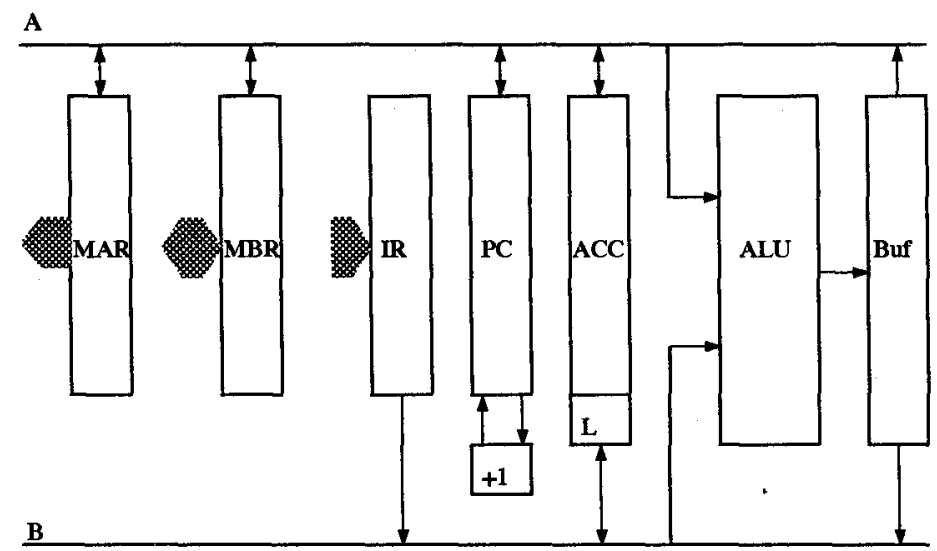

Fig. 1. The Datapath DP.

6.1. The Datapath. We will use the two-bus datapath, illustrated in Fig. 1. As well as the user-visible registers ACC, L and PC, there are four further registers, two buses $\mathrm{A}$ and $\mathrm{B}$, the ALU and an incrementer for PC. The memory address register MAR holds memory addresses, and is linked to the address bus. The memory buffer register MBR holds data to be read/written from/to memory, and is linked to the data bus. The instruction register IR holds the current instruction, and is linked separately to the data bus. The ALU buffer register is used as a store for ALU results. Additionally, the results of ALU test operations are available to the controller.

6.2. The Controller. We will use a microcoded controller $C T$ to control $D P$. To avoid confusion, we will use microinstruction to refer to instructions of $C T$, and instruction to refer to instructions of COMP. Each microinstruction will have three fields. The control field to operate $D P$; the sequencing field to determine the next microinstruction; and the address field to contain a microinstruction address.

The sequencing field will allow the following sequences: next sequential microinstruction; unconditional or conditional jump; unconditional or conditional subroutine; and decode next instruction. The last is used when the sequence of microinstructions to execute the current instruction ends, and a new instruction starts. Conditional jumps and subroutine calls are based on the results of ALU test operations. Also only one level of subroutine is allowed, as the return addresses are stored in a register. Fig. 2. illustrates the microprogrammed controller.

6.3. Formal Specification of DP and CT. We now consider the formal specification of $D P$ and $C T$ as iterated maps. We wish to construct an algebra

$$
\text { (Ct, Dp,Mem, } S \mid C T, D P, S M E M) \text {, }
$$

where $C t$ is the state of the controller $C T, D p$ is the state of the datapath $D P, M e m$ is the memory as in $\S 5, S$ is the system clock which is faster than instruction clock $T$, and $C T, D P$ and $S M E M$ are iterated maps representing 


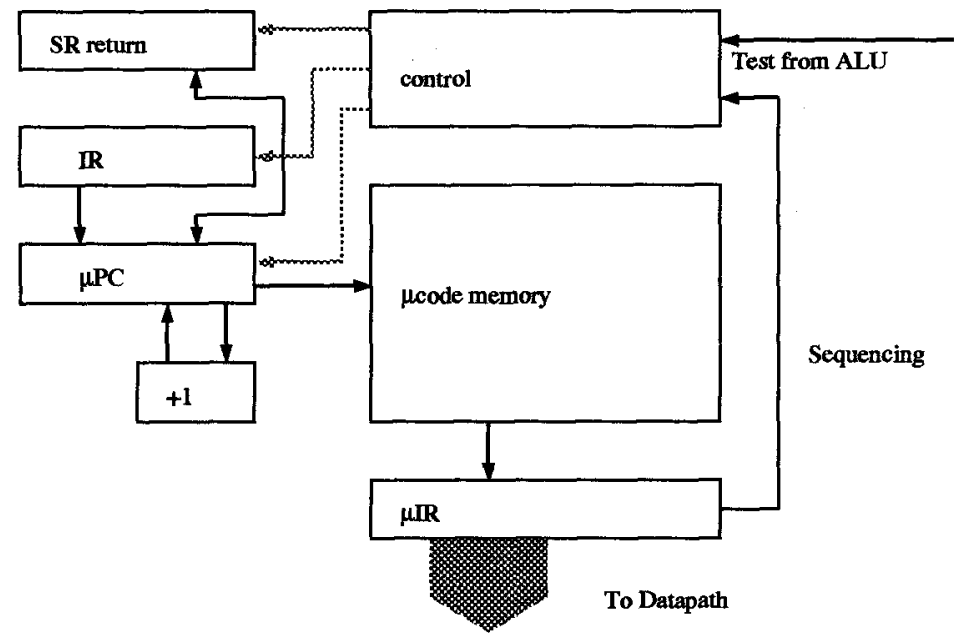

Fig. 2. The Controller.

$C T, D P$ and memory over clock $S$.

$$
\begin{aligned}
& C T: S \times C t \times D p \times M e m \rightarrow C t, \\
& D P: S \times C t \times D p \times M e m \rightarrow D p, \\
& S M E M: S \times C t \times D p \times M e m \rightarrow M e m,
\end{aligned}
$$

are defined by

$$
\begin{aligned}
& C T(0, c, d, m)=c, \\
& D P(0, c, d, m)=d, \\
& \operatorname{SMEM}(0, c, d, m)=m, \\
& C T(s+1, c, d, m)=\operatorname{ct}(C T(s, c, d, m), D P(s, c, d, m), \operatorname{SMEM}(s, c, d, m)), \\
& D P(s+1, c, d, m)=d p(C T(s, c, d, m), D P(s, c, d, m), \operatorname{SMEM}(s, c, d, m)), \\
& \operatorname{SMEM}(s+1, c, d, m)= \\
& \quad \operatorname{smem}(C T(s, c, d, m), D P(s, c, d, m), \operatorname{SMEM}(s, c, d, m)),
\end{aligned}
$$

To proceed, we must define state sets $C t$ and $D p$, as well as next-state functions $c t, d p$ and smem. Space does not permit the inclusion of definitions for $c t, d p$ and smem. However, the process is the same as that followed for PM in $\S 5$. First, we consider $D p$. Observe in fig. 1 that the state of $D P$ consists of six registers of size $A$ (ACC, PC, MAR, MBR, IR and ALUBUF), and the single-bit register $\mathrm{L}$. Additionally, there is a single-bit $A L U$ test register: Hence:

$$
D p=A \times A \times A \times A \times A \times B i t \times A \times B i t .
$$

Now, we consider $C t$. Observe in fig. 2 that the state of $C T$ consists of a microprogram counter $\mu P C$, a subroutine return address register $S R$, and the microcode memory. Let $[\mu P C \rightarrow \mu I R]$ be the microprogram memory. We can define $C t$ :

$$
C t=\mu P C \times S R \times[\mu P C \rightarrow \mu I R] .
$$


We construct the abstract circuit design (see §3.1) of the computer $\mu$ COMP in the manner of $\S 3.3$ (see also $\S 5.5$ ). We may define $\mu C O M P: S \times C t \times D p \times$ $M e m \rightarrow C t \times D p \times M e m$ as follows:

$$
\mu \operatorname{COMP}(s, c, d, m)=(C T(s, c, d, m), D P(s, c, d, m), \operatorname{SMEM}(s, c, d, m)) .
$$

\section{Correctness of the Implementation.}

For $\mu$ COMP (\$6.3) to be a correct implementation of COMP (\$5.5) the following diagram must commute:

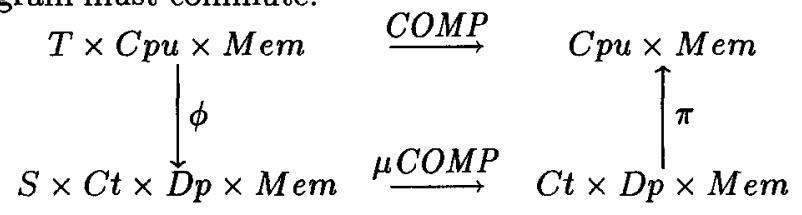

where $\pi: C t \times D p \times M e m \rightarrow C p u \times M e m$ is the following projection function

$\pi(\mu p c, s r, \mu m, a, p c, m a r, m b r, i r, l$, alubuf, alutest,$m)=(a, p c, l, m)$,

and $\phi: T \times C p u \times M e m \rightarrow S \times C t \times D p \times M e m$ is defined by

$$
\phi(t, c, m)=\left(\bar{\lambda}(t, c, m), \alpha_{x}(c, m)\right),
$$

where $\alpha_{x}: C p u \times M e m \rightarrow C t \times D p \times M e m$ is defined by

$$
\alpha_{x}(a, p c, l, m)=\left(x_{\mu p c}, x_{s r}, x_{\mu m}, a, p c, x_{m a r}, x_{m b r}, x_{i r}, l, x_{a l u b u f}, x_{a l u t e s t}, m\right),
$$

with $x_{\mu p c}, x_{s r}, x_{\mu m}, x_{m a r}, x_{m b r}, x_{i r}, x_{a l u b u f}, x_{\text {alutest }} \in x$. Note that the values in $x$ are not important except for the microcode memory $x_{\mu m}$.

It remains to define $\bar{\lambda}: T \times C p u \times M e m \rightarrow S$. Recalling $\$ 2.6$, we define a state-dependent retiming $\lambda: S \times C p u \times M e m \rightarrow T$ with $\bar{\lambda}$ as the immersion. We define XTIME :Cpu $\times$ Mem $\rightarrow\left[T \rightarrow \mathrm{N}^{+}\right]$, so that $\lambda(s, c, m)=$ $L(X T I M E(c, m))(s)$, where $L:\left[T \rightarrow \mathbf{N}^{+}\right] \rightarrow \operatorname{Ret}(S, T)$ is defined in $\S 2.6$. $X T I M E$ is defined by

$$
X T I M E(c, m)(t)=x \operatorname{time}(\operatorname{COMP}(t-1, c, m)) .
$$

The time taken for the instruction executed at time $t$ depends on the state of COMP at time $t-1$. Function xtime $: C p u \times M e m \rightarrow \mathbf{N}^{+}$determines how many cycles of clock $S$ the next instruction in state $c \in C p u, m \in M e m$ will take to execute.

$$
x \operatorname{time}(c, m)=(\text { least } s)\left[\pi_{\text {next }}\left(\mu \operatorname{COMP}\left(s, \alpha_{x}(c), m\right)\right)\right],
$$

where $\pi_{\text {next }}: C t \times D p \times M e m \rightarrow \mathbf{B}$ is a function that determines when execution of the current instruction is complete, by examining the appropriate part of the microinstruction word: see $\S 6.2$.

\section{Further Considerations.}

We have shown how computers may be algebraically modelled as iterated maps at different levels of abstraction, and defined what it means for a lower-level algebraic model to correctly implement a higher-level specification. Additionally, we have applied our algebraic tools to a case study.

The case study can be continued: both $P M$ and $A C$ can be completed, and the correctness condition verified. That is, does the diagram of $\$ 7$ commute? The process of modelling and verification of the components of the computer can continue with $D P$ and $C T$, each of which may be further subdivided. 


\section{References.}

G Birtwistle and B Graham, "Verifying SECD in HOL", pp.129-177 in Formal Methods for VLSI Design, ed. J Staunstrup, North-Holland, (1990).

A Cohn and M Gordon, "A Mechanised Proof of Correctness of a Simple Counter", pp.65-96 in Theoretical Foundations for VLSI Design, ed. K McEvoy and J V Tucker, Cambridge University Press, (1990).

A Cohn, "A Proof of Correctness of the VIPER Microprocessor: the First Levels", pp.27-72 in VLSI Specification, Verification and Synthesis, ed. G Birtwistle and P A Subrahmanyam, Kluwer Academic Publishers, (1987).

W J Cullyer, "Implementing Safety Critical Systems: the Viper Microprocessor", pp.1-26 in VLSI Specification, Verification, and Synthesis, ed. G Birtwistle and P A Subrahmanyam, Kluwer Academic Publishers, (1987).

W J Cullyer, "Application of Formal Methods to the VIPER Microprocessor", IEE Proceedings, 134 E, 3, pp.133-141 (May 1987).

J S Florentin, Microprogrammed Systems Design, Macmillan, (1991).

A Geser, "A Specification of the Intel 8085 Microprocessor: A Case Study.", pp.347-402 in Algebraic Methods: Theory, Tools and Applications, ed. M Wirsing and J A Bergstra, Lecture Notes in Computer Science 394, SpringerVerlag, (1989).

M Gordon, "Proving a Computer Correct with the LCF-LSM Hardware Verification System", Technical Report No. 42, Computer Laboratory, University of Cambridge, (1983).

M Gordon, "HOL: A Proof Generating System for Higher-Order Logic", pp.73-128 in VLSI Specification, Verification and Synthesis, ed. G Birtwistle and P A Subrahmanyam, Kluwer Academic Publishers, (1987).

B Graham and G Birtwistle, "Formalising the Design of an SECD Chip", pp.40-66 in Hardware Specification, Verification and Synthesis: Mathematical Aspects, ed. M Leeser and G Brown, Lecture Notes in Computer Science 408, Springer Verlag, (1990).

B Graham, The SECD Microprocessor: a Verification Case Study, Kluwer, (1992).

N A Harman and J V Tucker, "Clocks, Retimings, and the Formal Specification of a UART", pp.375-396 in The Fusion of Hardware Design and Verification, ed. G J Milne, North-Holland, (1988).

N A Harman and J V Tucker, "Formal Specification and the Design of Verifiable Computers", pp.500-503 in Proceedings of the 1988 UK IT Conference, University College Swansea, IEE, (1988).

N A Harman and J V Tucker, "The Formal Specification of a Digital Correlator I: Abstract User Specification", pp.161-262 in Theoretical Foundations for VLSI Design, ed. K McEvoy and J V Tucker, Cambridge University Press, (1990).

N A Harman and J V Tucker, "Consistent Refinements of Specifications for Digital Systems", pp.273-295 in Correct Hardware Design Methodologies, ed. P Prinetto and P Camurati, North-Holland, (1992).

N A Harman, "Formal Specifications for Digital Systems", Ph.D. Thesis, School of Computer Studies, University of Leeds, (1989).

W A Hunt, "FM8501: A Verified Microprocessor", The University of Texas at Austin Institute for Computing Science technical report 47, (1986).

S D Johnson and Z Zhu, "An Algebraic Approach to Hardware Specification and Derivation", in Proceedings of the IFIP International Workshop on Applied Formal Methods for Correct VLSI Design, ed. L Claesen, Elsevier, (1991).

J Joyce, "Formal Verification and Implementation of a Microprocessor", pp.129-159 in VLSI Specification, Verification and Synthesis, ed. G Birtwistle and P A Subrahmanyam, Kluwer Academic Publishers, (1987). 
P Landin, "On the Mechanical Evaluation of Expressions", Computer Journal, (1963).

K Meinke and J V Tucker, "Universal Algebra", pp.189-411 in Handbook of Logic in Computer Science, ed. S Abramsky, D Gabbay, T S E Maibaum, Oxford University Press, (1992).

T Melham, "Using Recursive Types to Reason about Hardware in Higher Order Logic", pp.27-50 in The Fusion of Hardware Design and Verification, ed. G J Milne, North-Holland, (1988).

G J Milne, "Timing Constraints: Formalising their Description and Verification", in Proceedings of Computer Hardware Description Languages and their Applications, North-Holland, (1989).

G J Milne, "The Formal Description and Verification of Hardware Timing”, University of Strathclyde Computer Science Report HDV-8-90, (1990).

W Stallings, Computer Organisation and Architecture: Principles of Function and Structure, Macmillan, (1987).

V Stavridou, Formal Specification of Digital Systems, Cambridge Tracts in Theoretical Computer Science, CUP (in press), (1993).

P A Subrahmanyam, "Contextual Constraints, Temporal Abstraction and Observational Equivalence in VLSI Design", pp.159-184 in The Fusion of Hardware Design and Verification, ed. G J Milne, North-Holland, (1988).

B C Thompson and J V Tucker, "Equational Specification of Synchronous Concurrent Algebras and Architectures", Department of Computer Science CSR 9.91, University College Swansea, (1991).

J V Tucker and J I Zucker, Program Correctness over Abstract Data Types with Error State Semantics, North-Holland, (1988).

J V Tucker and J I Zucker, "Generalised Computability and Algebraic Specifications for Abstract Data Types", In preparation, (1993).

J V Tucker, "Theory of Computation and Specification over Abstract Data Types and its Applications", in Logic, Algebra and Computation, ed. F L Bauer, Springer, (1991).

W Wechler, "Universal Algebra for Computer Scientists", EATCS Monograph, Springer-Verlag, Berlin, (1991.).

W P Weijland, "Verification of a Systolic Algorithm in Process Algebra", in Theoretical Foundations for VLSI Design, ed. K McEvoy and J V Tucker, Cambridge University Press, (1990).

M Wirsing, "Algebraic Specification", pp.675-788 in Handbook of Theoretical Computer Science, Volume B: Formal Models and Semantics, ed. J van Leeuwen, Elsevier, (1990).

Z Zhu and S D Johnson, "An Example of Interactive Hardware Transformation", Indiana University, Computer Science Department (draft), (1991). 